PROCEEDINGS OF THE

AMERICAN MATHEMATICAL SOCIETY

Volume 126, Number 2, February 1998, Pages 343-348

S $0002-9939(98) 04126-4$

\title{
SUBNORMAL SUBGROUPS OF GROUP RING UNITS
}

\author{
ZBIGNIEW S. MARCINIAK AND SUDARSHAN K. SEHGAL
}

(Communicated by Ronald M. Solomon)

\begin{abstract}
Let $G$ be an arbitrary group. If $a \in \mathbb{Z} G$ satisfies $a^{2}=0, a \neq 0$, then the units $1+a, 1+a^{*}$ generate a nonabelian free subgroup of units. As an application we show that if $G$ is contained in an almost subnormal subgroup $V$ of units in $\mathbb{Z} G$ then either $V$ contains a nonabelian free subgroup or all finite subgroups of $G$ are normal. This was known before to be true for finite groups $G$ only.
\end{abstract}

\section{INTRODUCTION}

Let $\mathbb{Z} G$ denote the integral group ring of a group $G$. Let $\mathcal{U}_{1} \mathbb{Z} G$ be the group of invertible elements in this ring which are of augmentation one: if $u=\sum u_{g} g$ then $\sum u_{g}=1$.

Let $G$ be an arbitrary group. Clearly $G \leq \mathcal{U}_{1} \mathbb{Z} G$. If not all finite subgroups of $G$ are normal in $G$ then we are able to construct a nontrivial unit $u \in \mathcal{U}_{1} \mathbb{Z} G, u \notin G$, in the following way. Choose $x, y \in G$ so that $o(x)=n\left\langle\infty\right.$ and $y\langle x\rangle y^{-1} \neq\langle x\rangle$. For $a=(1-x) y\left(1+x+\cdots+x^{n-1}\right) \in \mathbb{Z} G$ we have $a \neq 0$ and $a^{2}=0$. Hence $u=1+a$ is a unit and $u^{-1}=1-a$. Units obtained in this way are called bicyclic units.

It is well known that $\mathcal{U}_{1} \mathbb{Z} G$ quite often contains a nonabelian free subgroup. In fact, the following theorem was proved by Sehgal [13, p. 200] and also by HartleyPickel [4] (see also [14, p. 19]).

0.1. Theorem. Let $G$ be a solvable group which has a non normal finite subgroup. Then $\mathcal{U}_{1} \mathbb{Z} G$ contains a nonabelian free subgroup.

Also, all nonabelian finite groups $G$, except Hamiltonian 2-groups, have a free subgroup inside $\mathcal{U}_{1} \mathbb{Z} G$.

Free subgroups in $\mathcal{U}_{1} \mathbb{Z} G$ were also studied by Jespers who characterized in [5] all finite groups $G$ which have a free complement in $\mathcal{U}_{1} \mathbb{Z} G$. Jespers, Leal and del Río classified in [6] all finite nilpotent groups $G$ such that $\mathcal{U}_{1} \mathbb{Z} G$ has a subgroup of finite index which is a direct product of noncyclic free groups. Recently Leal and del Río [7] removed the nilpotent condition and classified all finite groups with the same property.

These results were obtained by a careful study of rational representations of finite groups. Except a few examples, these were really existence results.

Received by the editors August 11, 1996.

1991 Mathematics Subject Classification. Primary 16S34, 16 U60.

This research was supported by Canadian NSERC Grant A-5300 and Polish Scientific Grant 2P30101007.

(C) 1998 American Mathematical Society 
Recently, we have found an elementary and explicit construction of a free subgroup inside $\mathcal{U}_{1} \mathbb{Z} G$, also for infinite groups. Namely, we proved in [10] that if $u \in \mathbb{Z} G$ is a bicyclic unit then $\left\langle u, u^{*}\right\rangle$ is a nonabelian free subgroup of $\mathcal{U}_{1} \mathbb{Z} G$, where $\left(\sum a_{g} g\right)^{*}=\sum a_{g} g^{-1}$.

Also assuming the existence of bicyclic units, Goncalves and Passman gave in [3] a construction of a free subgroup inside $\mathcal{U}_{1} K G$ when $K$ is a field, $\operatorname{char} K=p>0$, tr.deg. $K>0$ and $G$ has no $p$-torsion.

In the present paper we extend the main result of [10] in the following way.

0.2. Theorem. Let $a \in \mathbb{Z} G$ be an arbitrary element satisfying $a \neq 0, a^{2}=0$. Set $u=1+a$. Then the subgroup $\left\langle u, u^{*}\right\rangle<\mathcal{U}_{1} \mathbb{Z} G$ is free and nonabelian.

We apply this theorem to generalize the main result of [2]. Let us say that a subgroup $V<\mathcal{U}_{1} \mathbb{Z} G$ is almost subnormal if $V$ is subnormal in a finite index subgroup of $\mathcal{U}_{1} \mathbb{Z} G$.

0.3. Theorem (Goncalves-Ritter-Sehgal, [2]). Let $G$ be a finite group. If $V<$ $\mathcal{U}_{1} \mathbb{Z} G$ is an almost subnormal subgroup containing $G$ then either

(i) $V$ has a nonabelian free subgroup, or

(ii) $G$ is abelian or a Hamiltonian 2-group.

The original proof depended on the classification of finite groups all of whose proper subgroups and factor groups are Hamiltonian 2-groups or abelian, followed by a careful investigation of their rational representations.

Notice that all subgroups of groups mentioned in (ii) are normal. Keeping this in mind, we offer the following generalization:

0.4. Theorem. Let $G$ be an arbitrary group. If $V<\mathcal{U}_{1} \mathbb{Z} G$ is an almost subnormal subgroup containing $G$ then either

(i) $V$ has a nonabelian free subgroup, or

(ii) all finite subgroups of $G$ are normal.

\section{Free subgroups in $\mathcal{U}_{1} \mathbb{Z} G$}

We construct here some free subgroups inside $\mathcal{U}_{1} \mathbb{Z} G$. In fact, it is equally easy to do this in a more general context.

Let $A$ be a $\mathbb{C}$-algebra with an involution denoted by $*$. Following $[12$, p. 302] we say that a Hermitian inner product $(-,-): A \times A \longrightarrow \mathbb{C}$ is compatible with $*$ if $\left(a^{*} b, c\right)=(b, a c)$ holds for all $a, b, c \in A$.

1.1. Examples. (a) Let $A=\mathbb{C} G$, the group algebra of an arbitrary group $G$ with the standard involution $\left(\sum a_{g} g\right)^{*}=\sum \bar{a}_{g} g^{-1}$. The Hermitian inner product compatible with $*$ is defined by $(a, b)=\operatorname{tr}\left(a^{*} b\right)$ where tr is the group ring trace: $\operatorname{tr}\left(\sum a_{g} g\right)=a_{1}($ compare $[11$, p.33]).

(b) Let $A=M_{n}(\mathbb{C})$, the matrix ring with the involution $a^{*}=\bar{a}^{T}$. The Hermitian inner product compatible with $*$ is given by $(a, b)=\operatorname{Tr}\left(a^{*} b\right)$ where $\operatorname{Tr}$ is the usual matrix trace.

(c) Let $A$ be any $H^{*}$-algebra. It is a Banach $*$-algebra whose norm is a Hilbert space norm such that $\left(a, b c^{*}\right)=(a c, b)=\left(c, a^{*} b\right)$ for all $a, b, c \in A$ (see [1]).

We can equip any such algebra with a norm $\|\cdot\|: A \longrightarrow \mathbb{R}$ defined by $\|a\|=$ $(a, a)^{1 / 2}$. 
The main result of this section is

1.2. Theorem. Let $A$ be a $\mathbb{C}$-algebra with an involution $*$ and with a compatible Hermitian inner product (-,-). If an element $a \in A$ satisfies $a^{2}=0$ and $\left\|a^{*} a\right\| \geq$ $4 \cdot\|1\|$ then $\left\langle 1+a, 1+a^{*}\right\rangle$ is a free nonabelian subgroup of units in $A$.

For the proof we consider the sequence $T=\left\{1, c, c^{2}, c^{3}, \ldots\right\}$ of elements in $A$, where $c=a^{*} a \in A$. In what follows we will assume that $\|c\| \geq 4 \cdot\|1\|$. Our argument splits into two cases depending on the linear dependence of $T$.

1.3. Lemma. If the sequence $T$ is linearly independent then

(i) the submonoid $S$ of $(A, \cdot)$ generated by $\left\{a, a^{*}\right\}$ is isomorphic to $\langle s, t| s^{2}=0=$ $\left.t^{2}\right\rangle$,

(ii) the linear span of $S$ in $A$ is isomorphic to the (reduced) semigroup algebra $\mathbb{C} S$.

Proof. Let $\Sigma$ be the monoid given by the presentation $\left\langle s, t \mid s^{2}=0, t^{2}=0\right\rangle$. Consider the monoid map $\phi: \Sigma \longrightarrow S$ given by $\phi(s)=a^{*}, \phi(t)=a$.

To prove (i) it is enough to show that $\phi$ is injective. To this end we decompose $\Sigma$ into five pairwise disjoint pieces: $\Sigma=\{0\} \cup \mathcal{T} \cup t \mathcal{T} \cup \mathcal{T} s \cup t \mathcal{T} s$, where $\mathcal{T}=$ $\left\{(s t)^{k} \mid k \geq 0\right\}$. Notice that $\phi$ is injective on $\mathcal{T} \subset \Sigma$ as, by assumption, all powers of $c$ are distinct.

Suppose that $\phi(x)=\phi(y)$ for two distinct elements $x, y \in \Sigma \backslash \mathcal{T}$. If $x, y$ belong to the same part of $\Sigma$, we can multiply them both on the left by $s$ and/or on the right by $t$ to obtain two distinct elements of $\mathcal{T}$ mapping to the same element of $S$. This is a contradiction with the last paragraph. On the other hand, if $x, y$ belong to different parts of $\Sigma$ then a simultaneous left and/or right multiplication gives us a new pair $x^{\prime}=0, y^{\prime} \in \mathcal{T}$. But then $0=\phi\left(x^{\prime}\right)=\phi\left(y^{\prime}\right) \in T$ - a contradiction, as $T$ is linearly independent. This proves (i).

To prove (ii) it is enough to show that $S \backslash\{0\}$ is linearly independent in $A$. Consider any linear combination of elements from $S \backslash\{0\}$. We can write it in the form $\alpha+\beta+\gamma+\delta=0$ where the four summands are combinations of elements from $T, a T, T a^{*}$ and $a T a^{*}$ respectively. Note that these sets cover $S \backslash\{0\}$ and are pairwise disjoint by part (i).

If $\alpha \not \equiv 0$ then we multiply our combination on both sides by $a^{*} a$. We obtain a non-trivial combination $\left(a^{*} a\right) \alpha\left(a^{*} a\right)=0$ of elements from $T$, a contradiction. Hence $\alpha \equiv 0$ and so $\beta+\gamma+\delta=0$.

We can continue in this way to show that also $\beta \equiv \gamma \equiv \delta \equiv 0$. For details look in the proof of Lemma 6 in [10].

1.4. Lemma. If the sequence $T$ is linearly dependent then the linear map $f: A \longrightarrow$ $A$ given by $f(x)=c x$ has an eigenvalue $\lambda$ with $|\lambda| \geq 4$.

Proof. Choose a maximal positive integer $d$ so that the set $T_{d}=\left\{1, c, \ldots, c^{d-1}\right\}$ is linearly independent. Let $W$ be the $\mathbb{C}$-linear span of $T_{d}$ in $A$. Then $c^{d} \in W$ and hence the finite dimensional subspace $W$ is invariant under $f$.

Notice that $c^{*}=c$. Then for $v, w \in W$ we have $(v, f(w))=(v, c w)=\left(c^{*} v, w\right)=$ $(c v, w)=(f(v), w)$, i.e. $f: W \longrightarrow W$ is a Hermitian operator with respect to the Hermitian inner product $(-,-)_{\mid W}$. From the Spectral Theorem (see [9, Ch.XIV, §12]) it follows that $W$ has an orthonormal basis $v_{1}, \ldots, v_{d}$ consisting of eigenvectors of $f$. 
Let $f\left(v_{i}\right)=\lambda_{i} v_{i}$ for $i=1, \ldots, d$. We prove that we must have $\left|\lambda_{i}\right| \geq 4$ for at least one $i$. To this end write $1 \in W \subset A$ as a combination of the eigenvectors: $1=\sum \alpha_{i} v_{i}, \alpha_{i} \in \mathbb{C}$. Then $c=c \cdot 1=\sum \alpha_{i} c v_{i}=\sum \alpha_{i} f\left(v_{i}\right)=\sum \alpha_{i} \lambda_{i} v_{i}$. Hence $\|c\|^{2}=(c, c)=\sum\left|\alpha_{i} \lambda_{i}\right|^{2}$, by the Pythagorean Theorem.

Suppose that $\left|\lambda_{i}\right|<4$ holds for $i=1, \ldots, d$. Then $\|c\|^{2}<16 \cdot \sum\left|\alpha_{i}\right|^{2}=16 \cdot\|1\|^{2}$ and so $\|c\|<4 \cdot\|1\|-$ a contradiction with the assumption that $\|c\| \geq 4 \cdot\|1\|$.

Proof of Theorem 1.2. We look at the sequence $T$. If $T$ is linearly independent then, by Lemma 1.3, the subalgebra $R \subset A$ generated by $\left\{a^{*}, a\right\}$ is isomorphic to the semigroup algebra $\mathbb{C} \Sigma$. Consider the monoid map $\rho: \Sigma \longrightarrow M_{2}(\mathbb{C})$ given by $\rho(s)=\left(\begin{array}{l}0 \\ 0\end{array}\right), \rho(t)=\left(\begin{array}{ll}0 & 0 \\ 2 & 0\end{array}\right)$. We extend $\rho$ by linearity to an algebra homomorphism

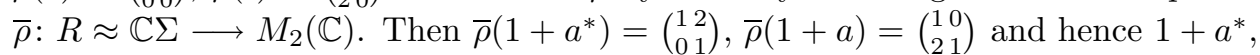
$1+a$ generate together a nonabelian free subgroup, by a well known theorem of Sanov:

1.5. Theorem (Sanov, see $[8$, p. 92]). For any complex number $z$ satisfying $|z| \geq$

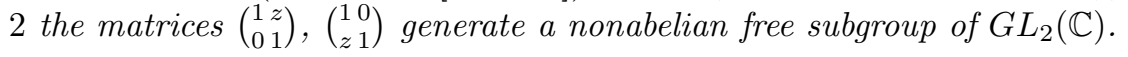

We are thus left with the case when $T$ is linearly dependent. By Lemma 1.4 there exists an element $v \in A, v \neq 0$, such that $a^{*} a \cdot v=\lambda v$ and $|\lambda| \geq 4$. Choose $\mu \in \mathbb{C}$ so that $\mu^{2}=\lambda$ and define $w=(1 / \mu) a \cdot v \in A$.

The subspace $V=\operatorname{span}_{\mathbb{C}}(v, w) \subset A$ is invariant under left multiplication by $a^{*}$ and by $a$. In fact,

$$
\begin{array}{ll}
a^{*} \cdot v=(1 / \lambda)\left(a^{*}\right)^{2} a v=0, & a \cdot v=\mu w \\
a^{*} \cdot w=(1 / \mu) a^{*} a v=\mu v, & a \cdot w=(1 / \mu) a^{2} v=0 .
\end{array}
$$

From the above equalities it follows that the vectors $v, w$ are linearly independent and hence they form a basis of $V$.

Obviously, left multiplications by $1+a^{*}$ and $1+a$ also preserve the subspace $V$. With respect to the basis $\{v, w\}$ they are represented by the matrices

$$
1+a^{*} \leftrightarrow\left(\begin{array}{cc}
1 & \mu \\
0 & 1
\end{array}\right), \quad 1+a \leftrightarrow\left(\begin{array}{cc}
1 & 0 \\
\mu & 1
\end{array}\right)
$$

However, we have $|\lambda| \geq 4$ and hence $|\mu| \geq 2$. Therefore the above pair of matrices generates a free group, by Theorem 1.5. Hence the units $1+a^{*}, 1+a \in A$ do the same.

Now we specialize to group algebras.

1.6. Theorem. If an element $a \in \mathbb{C} G$ satisfies $a^{2}=0$ and $\|a\| \geq 2$ then $\langle 1+a$, $\left.1+a^{*}\right\rangle$ is a free nonabelian subgroup of units in $\mathbb{C} G$.

Proof. Let $c=a^{*} a$. Then we have $\|c\|^{2}=(c, c)=\operatorname{tr}\left(c^{*} c\right)=\sum\left|c_{g}\right|^{2} \geq\left|c_{1}\right|^{2}$. It follows that $\|c\| \geq\left|c_{1}\right|=|\operatorname{tr}(c)|=\left|\operatorname{tr}\left(a^{*} a\right)\right|=|(a, a)|=\|a\|^{2} \geq 4$. Hence $\left\|a^{*} a\right\| \geq 4=4 \cdot\|1\|$ and we may apply Theorem 1.2.

1.7. Theorem. If an element $a \in \mathbb{Z} G$ satisfies $a^{2}=0$ and $a \neq 0$ then $\left\langle 1+a, 1+a^{*}\right\rangle$ is a free nonabelian subgroup of units in $\mathbb{Z} G$.

Proof. By the previous theorem it is enough to show that $\|a\| \geq 2$. Suppose then that $\|a\|<2$, i.e. $4>\|a\|^{2}=\sum a_{g}^{2}$. Hence all $a_{g}$ belong to $\{-1,0,1\}$ and $a$ has at most three elements in its support. From $a^{2}=0$ it follows that $a$ has augmentation zero and hence $a=g-h$ for some $g, h \in G$. Then from $0=a^{2}=g^{2}+h^{2}-g h-h g$ it easily follows that $g=h$ and $a=0-$ a contradiction. 
1.8. Corollary. If $\mathbb{Z} G$ contains a nonzero nilpotent element then the unit group of $\mathbb{Z} G$ contains a nonabelian free subgroup.

1.9. Corollary. Suppose that the group ring $\mathbb{Z} G$ is not a domain. If $G$ has no nonidentity finite normal subgroups then the group of units of $\mathbb{Z} G$ contains a nonabelian free subgroup.

Proof. Let $x, y \in \mathbb{Z} G$ be nonzero elements such that $x y=0$. From Connell's Theorem [11, Thm. 4.2.10] it follows that the algebra $\mathbb{Q} G$ is prime and hence $y \mathbb{Q} G x \neq 0$. Pick an element $z \in \mathbb{Z} G$ so that $a=y z x \neq 0$. Then $a^{2}=0$ and Theorem 1.7 applies.

\section{An Application}

Here we prove Theorem 0.4. We start with

2.1. Lemma. Suppose that an almost subnormal subgroup $V<\mathcal{U}_{1} \mathbb{Z} G$ contains $G$. If $\mathbb{Z} G$ has a bicyclic unit then $V$ contains a nonabelian free subgroup.

Proof. Write $\mathcal{U}=\mathcal{U}_{1} \mathbb{Z} G$ for short. We have

$$
G<V=V_{r} \triangleleft V_{r-1} \triangleleft \cdots \triangleleft V_{1}<\mathcal{U}
$$

and $\left|\mathcal{U}: V_{1}\right|<\infty$. It follows there exists a normal subgroup $N \triangleleft \mathcal{U}$ such that $N<V_{1}$ and $|\mathcal{U}: N|<\infty$. Consider $N^{*}=\left\{u \in \mathcal{U} \mid u^{*} \in N\right\}$. Then also $\left|\mathcal{U}: N^{*}\right|<\infty$ and $N^{*} \triangleleft \mathcal{U}$. Set $\widetilde{N}=N \cap N^{*}$. Then we have $\widetilde{N}^{*}=\widetilde{N},|\mathcal{U}: \widetilde{N}|<\infty, \widetilde{N} \triangleleft \mathcal{U}$ and $\widetilde{N}<V_{1}$.

Let $u$ be a bicyclic unit and let $k$ be the order of the image of $u$ in $\mathcal{U} / \widetilde{N}$. Then $u^{k} \in \widetilde{N}$ and $\left(u^{*}\right)^{k}=\left(u^{k}\right)^{*} \in \widetilde{N}^{*}=\widetilde{N}$.

By construction $u=1-a$ where $a=(x-1) y\left(1+x+\cdots+x^{n-1}\right)$ for suitable $x, y \in G$. Consider the group ring elements $a_{s}=k(x-1)^{s} y\left(1+x+\cdots+x^{n-1}\right)$ for $s \geq 1$. Clearly $a_{s}^{2}=0$. Hence we have new units $u_{s}=1-a_{s} \in \mathcal{U}$.

Notice that $x u_{s} x^{-1}=1-x a_{s}$. It follows that $u_{s}=\left[x, u_{s-1}\right]$ for all $s \geq 2$. In fact,

$$
\begin{aligned}
{\left[x, u_{s-1}\right] } & =x \cdot\left(1-a_{s-1}\right) \cdot x^{-1} \cdot\left(1+a_{s-1}\right)=\left(1-x a_{s-1}\right)\left(1+a_{s-1}\right) \\
& =1+a_{s-1}-x a_{s-1}=1-(x-1) a_{s-1}=1-a_{s}=u_{s} .
\end{aligned}
$$

Finally, we show that $u_{s}, u_{s}^{*} \in V_{s}$ for all $s \geq 1$. Clearly $u_{1}=u^{k}$ and hence $u_{1}, u_{1}^{*} \in \widetilde{N}<V_{1}$. Assume that $u_{s-1} \in V_{s-1}$. Then $u_{s-1} x^{-1} u_{s-1}^{-1} \in V_{s}$ as $x^{-1} \in$ $G<V_{s} \triangleleft V_{s-1}$. Then also $u_{s}=x \cdot u_{s-1} x^{-1} u_{s-1}^{-1} \in V_{s}$. The proof that $u_{s}^{*} \in V_{s}$ is similar.

Now, by Theorem 1.7, $\left\langle u_{r}, u_{r}^{*}\right\rangle=\left\langle 1+a_{r}, 1+a_{r}^{*}\right\rangle\left\langle V_{r}=V\right.$ is a free subgroup.

Proof of Theorem 0.4. If $G$ has a finite subgroup which is not normal then $\mathcal{U}_{1} \mathbb{Z} G$ contains a bicyclic unit. From Lemma 2.1 it then follows that the subgroup $V$ contains a nonabelian free subgroup.

2.2. Corollary. Let $G$ be an arbitrary group. If $\mathcal{U}_{1} \mathbb{Z} G$ contains a subgroup $V$ such that

(i) $V$ is subnormal in a finite index subgroup of $\mathcal{U}_{1} \mathbb{Z} G$,

(ii) $G \leq V$,

(iii) $V$ is nilpotent

then all finite subgroups of $G$ are normal. 
2.3. Remark. Clearly, we could change nilpotent in the last corollary to solvable or any other nontrivial variety of groups. The proof remains the same.

\section{REFERENCES}

1. Ambrose, W., Structure theorems for a special class of Banach algebras, Trans. Amer. Math. Soc. 57 (1945), 364-386. MR 7:126c

2. Goncalves, J., Ritter, J., Sehgal, S.K., Subnormal subgroups in $\mathcal{U}_{1} \mathbb{Z} G$, Proc. Amer. Math. Soc. 103 (1988), 375-382. MR 89h:20006

3. Goncalves, J., Passman, D.S., Construction of free groups in the group of units of modular group algebras, Comm. Algebra 24 (1996), no. 13, 4211-4215. MR 97g:16043

4. Hartley, B., Pickel, P.F., Free subgroups in the unit groups of integral group rings, Canadian Journal of Math. 32 (1980), 1342-1352. MR 82i:20008

5. Jespers, E., Normal complements and the unit group of integral group rings, Proceedings of AMS 122 (1994), 59-66. MR 94k:16058

6. Jespers, E., Leal, G., del Río, A., Products of free groups in the unit group of integral group rings, to appear in J.Algebra.

7. Leal, G., del Río, A., Products of free groups in the unit group of integral group rings II, to appear in J. Algebra.

8. Kargapolov, M., Mierzljakov, Yu., Fundamentals of the theory of groups, Springer Verlag, 1979. MR 80k:20002

9. Lang, S., Algebra, Addison-Wesley Student Series, second printing 1970. MR 33:5416

10. Marciniak, Z. S., Sehgal, S. K., Constructing Free Subgroups of Integral Group Ring Units, Proc. Amer. Math. Soc. 125 (1997), 1005-1009. MR 97f:16057

11. Passman, D.S., Algebraic structure of group rings, Interscience, New York, 1977. MR 81d:16001

12. Rowen, L.H., Ring theory, Academic Press, 1988. MR 89h:16001; MR 89h:16002

13. Sehgal, S.K., Topics in group rings, Macel Dekker, 1978. MR 80j:16001

14. Sehgal, S.K., Units in integral group rings, Longman's, Essex, 1993. MR 94m:16039

Institute of Mathematics, Warsaw University, ul. Banacha 2, 02-097 Warszawa, POLAND

E-mail address: zbimar@mimuw.edu.pl

Department of Mathematical Sciences, University of Alberta, Edmonton, Canada T6G 2G1

E-mail address: S.Sehgal@ualberta.ca 